\title{
A scale for architectural design compactness: a mathematical approach
}

\author{
M. M. Saeed \\ Architectural Department, College of Engineering, \\ University of Salahaddin, Iraq
}

\begin{abstract}
The topic of compactness is significantly connected to sustainability, due to its impact in understanding and managing the process of shape-space formation, which may lead to an energy efficient design. This paper is a complement to a research delivered to a scientific conference of the Iraqi University of Technology in 1999 by the same researcher, discussing the concept of architectural design compactness, its prospects and development, the recent paper distinguishes between the shape compactness and the design compactness, and suggests a scale for the latter. The methodology adopted in this paper is, after defining the function of the architectural design compactness, reviewing all the relevant available scales and discussing their function to find whether they are sufficient to measure the design compactness. Discovering the contradictions of these scales results, lead to the necessity of deriving an objective and quantitative scale for the latter which is the aim of this paper. By analyzing the function of the architectural design compactness as a dependent variable, the independent variables which the latter relatively varies accordingly were devised, and then the scale is mathematically derived. Reliability of the scale has been verified by comparing the computed design compactness values of various building systems with their subjective evaluation; it shows a remarkable consistence with them.

A model of abstract plans representing various design systems is prepared to evaluate their compactness to be used as guidelines for designers.

The derived scale can be developed in a subsequent paper to establish a system for evaluating the compactness of multi-storey buildings by taking into account the rate of change for all external surfaces (walls and roofs).

Keywords: architectural design, compactness, shape factor, perimeter, surface area, volume, space, form.
\end{abstract}




\section{Introduction}

Architectural design compactness is one of the powerful tools that can be exploited to design energy efficient buildings meeting sustainability requirements. It can control the architectural design process, and also can be the complementary aspect to the evaluation of heat resistance of building components for controlling heat loss and gain. Compactness - in contrast to looseness - in the architectural design describes the degree of joining and attachment of building spaces. Buildings are considered compact when the shared sides of internal spaces (and with adjoining buildings) increase, and not only when the perimeter decreases, and considered loose when the shared sides of internal spaces decrease and not only when exposed sides with the external open space increase. Although there are many scales available for measuring the compactness of shapes or solids form, there is a lack of a quantitative scale for measuring architectural design compactness which if measured by the abstract shape and form scales may lead to a misunderstanding and contradiction of the architectural design evaluation.

In this paper, the proposed scale deals with single storey architectural systems, which consist of rectangular spaces connected to each other due to their attachment and sharing their sides partially or completely with others and with the external open spaces.

\section{Methodology}

\subsection{Review of the preceding studies}

Although compactness of architectural design has not been studied as an independent subject, many researchers referred to, when studying environmental design, or cost analysis of building enclosure, explained its effect, and concluded that the external perimeter of building varies according to the building shape and that a compact building is one that has a maximum floor area with a minimum perimeter. Blackwell [1] put the first step toward compactness by studying the properties of abstract geometrical shapes and their area/ perimeter ratios, for various sides proportion varying from (1) to (100). To explain the variation of perimeter relative to the side proportion, Blackwell did not use the absolute value of area/ perimeter ratio, but he related it to the same ratio of a circle of similar area to get a dimensionless value representing the degree of compactness for a shape and presented it graphically (fig. 1).

Evans [2], Burberry [3] and Vandenberg [4] went a farther step by introducing models representing the variation of perimeter relative to the compactness of the shape. The first, presented a model (fig. 2) of nine abstract forms, five of them single storey residential units of equal area but of different shapes, he compared the value of their exposed surface area to show their variation relative to shape when the floor area is constant. The second, plot a graph of three lines representing the heat loss ratios for three houses of $100 \mathrm{~m}^{2}$ area, and of 1:1, 1:2 and 1:3 sides proportion (fig. 3), and he explained the reasons for dissimilarity 
of heat loss, due to the unequal surface area of the three cases. The latter presented five abstract forms (fig. 4), two of them of equal area but of different shapes, he compared their wall to floor area to show the effect of shape on cost.

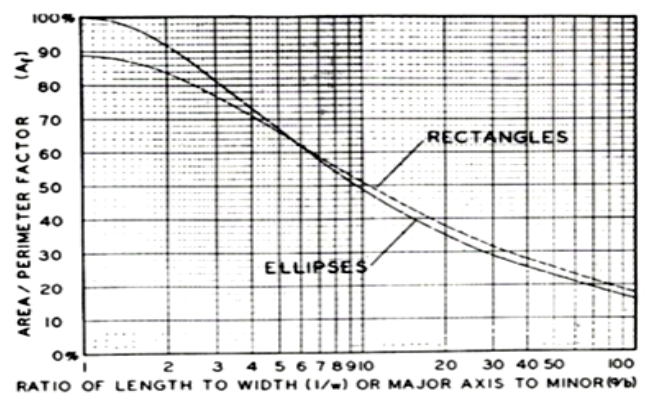

Figure 1: Area/perimeter factor relative to sides proportion.
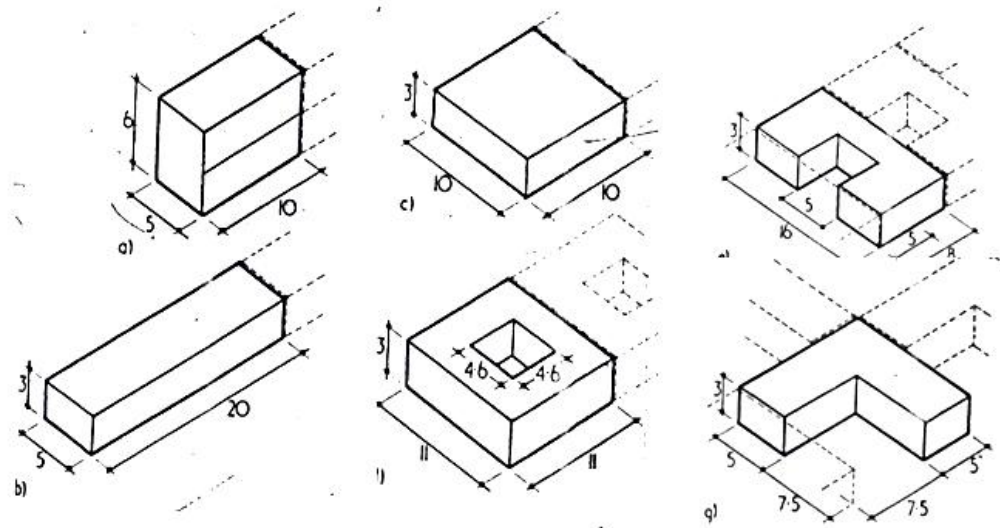

Figure 2: $\quad$ Variation of exposed surface area relative to shape.

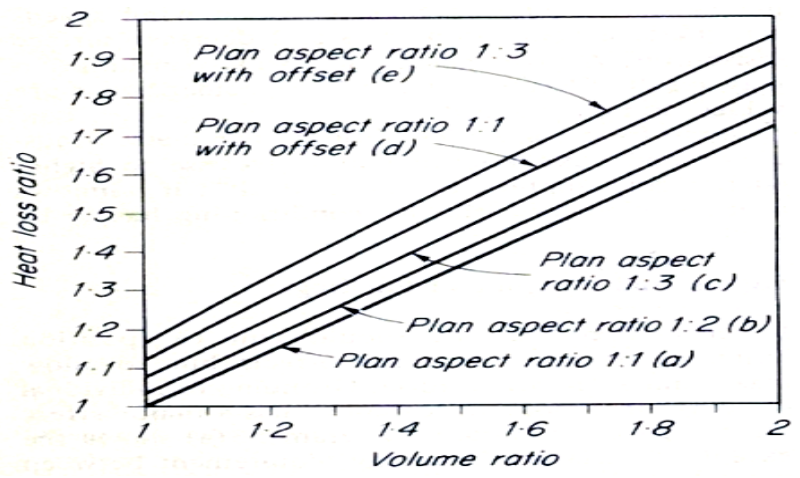

Figure 3: Variation of heat loss relative to aspect ratio. 


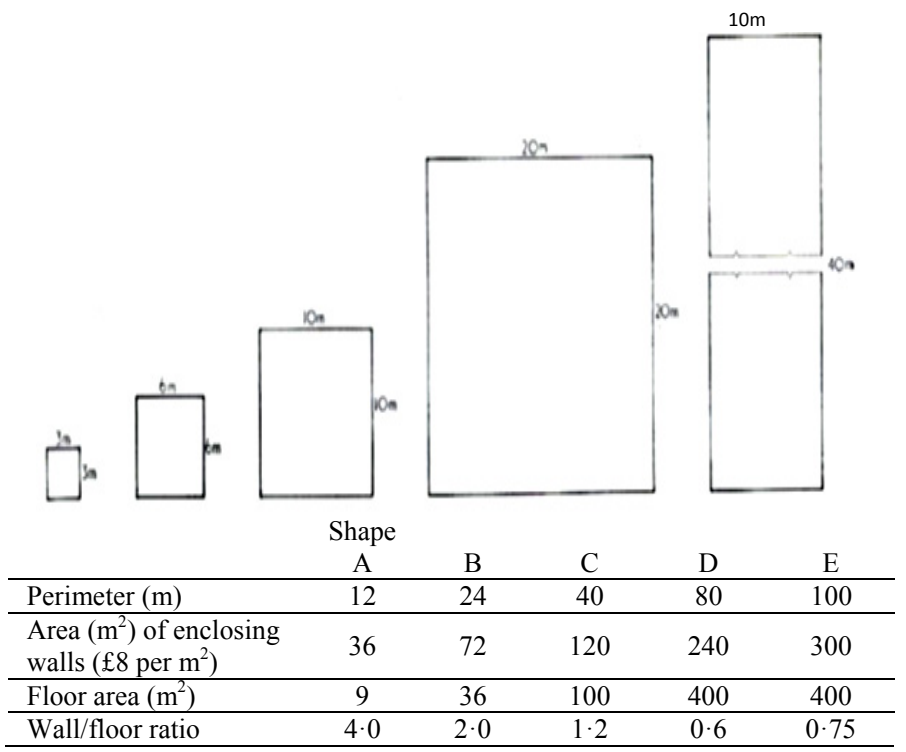

Figure 4: Variation of wall cost relative to shape.

Lechner [5] refer to another variable effecting the value of compactness; the number of units contained in a system, by presenting a model of four building blocks (fig. 5) for houses of equal areas and perimeter but each block contain different number of houses, he measured the average external exposed surface area of each house, for the four blocks and the saving in external walls due to the variation of the walls attaching the external space.

All the presented studies had not defined values of design compactness to any model due to the lack of a scale.
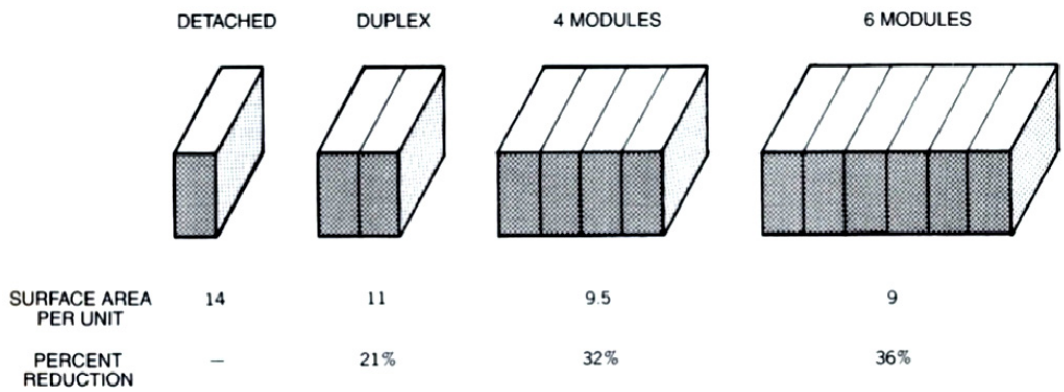

Figure 5: Variation of external wall cost relative to the number of units in a block. 
Saeed [6] explained the necessity of a scale for measuring the compactness of the architectural design to indicate the degree of joining and attachment of the building spaces due to their pattern of arrangement, within the surface or a volume and developed a model measuring the variation of design compactness as a dependent variable - in relative to all internal components perimeters and inversely with the sum of the exposed sides of the final shape formed by these components, as independent variables.

\subsection{Reviewing the available compactness scales, factors and ratios}

Shape factor is an independent orientation, dimensionless number and agree with intuitive notions of what makes a shape compact [7]. Many scales, factors and ratios have been derived to describes compactness of shapes and forms, some of these are dimensional with units of (1/length), such as; Perimeter / Floor area ratio, or External envelope/Volume, nominated as Coefficient of Compactness which is measured by the equation; $\mathbf{k}_{\mathbf{e}}=\mathbf{A} / \mathbf{V}_{\mathbf{e}} \mathrm{m}^{-1}[8-10]$. These scales can be used only to evaluate alternatives of a single system when one of the variables is constant, such as perimeter or floor area for the first scale, and $\mathbf{A}$ or $\mathbf{V} \mathbf{e}$ for the second. These factors cannot be considered as general scales due to their lack of the condition of being dimensionless numbers. Other scales such as; Wall / Floor area, External envelope / Floor area [4], although they are dimensionless, but still have the same lack of limited evaluation extent to a specific case, similar to the a.m. scales. Form factor derived by Mahdavi [11] or relative compactness as he nominated it- by his equation: $\mathrm{RC}=6 \frac{V^{2 / 3}}{A}$ can be an adequate measure for form compactness but not the design compactness.

Generally, all these scales are sharing the attribute of measuring the variation of perimeter of a shape relative to its area, or the variation of surface area relative to its volume, in other words they are describing the shape of the surface, or the form of the volume, regardless the arrangement of the components of these surfaces or volumes, if they are loose or compact.

These scales will face a contradiction in their results if intended to measure design compactness due to the fact that, well compacted solid (evaluated by the shape factor) have not necessarily a compacted arrangement of cells, the reverse is true too, less compacted solid can contain well compacted arrangement of cells.

To present an example explaining this fact, assume three volumes containing cubical subdivision; A, B and C (fig. 6 ), $\mathrm{A}$ is the biggest in plan area, divided into four equal cubes, B has the same number of cubes as in A but smaller, $\mathrm{C}$ is similar in area to B but divided into nine equal cubes. All scales devoted to describe shape compactness will show higher value for $\mathrm{A}$ than $\mathrm{B}$ and $\mathrm{C}$, because it has more unit volume per unit of surface area than the smaller ones, and equal values for $\mathrm{B}$ and $\mathrm{C}$ because both have similar unit volume per unit of surface area. While according to the explained design compactness function, compactness value of $\mathrm{A}$ is equal to $\mathrm{B}$, because cells in both volumes are compactness of $\mathrm{C}>\mathrm{A}$ and $\mathrm{B}$ because cells in $\mathrm{C}$ are more attaching each other internally and less exposed to the external space than $\mathrm{A}$ and $\mathrm{B}$. 

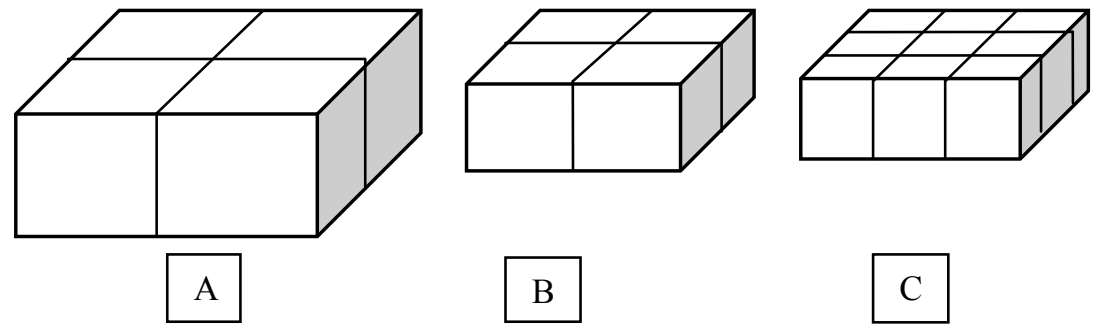

Figure 6: Comparison of compactness for three volumes containing cubical subdivision.

\subsection{Definition and variables of the architectural design compactness}

This paper distinguish between two different facts; shape compactness (nominated later as the shape factor) and the architectural design compactness, although both can measure two dimensional surfaces and three dimensional solids. Shape factor for two dimensional shapes is an abstract measure describing the degree of contract, shortening or elongation of a surface, and it is derived by comparing its perimeter to area ratio to that of the most compact shape with the same area; the square, if the circle shape excluded. While the architectural design compactness scale deals with plans of various shapes consisting of several internal cells (spaces), hence its value is associated significantly with the pattern of arrangement of these cells that establish the final shape and the degree of attachment between the cells and with the outdoor open space. Hence the architectural design compactness as a dependent variable varies relatively to the sum of components (cells) perimeter and inversely to the exposed sides of the final shape formed by these components, as independent variables, hence:

$$
C \propto \frac{\sum P_{n}}{P_{0}}
$$

where $C=$ architectural design compactness for a system.

$\sum P_{n}$ is the sum of components perimeter $=P_{1}+P_{2}+\ldots P_{n}$

$P_{0}=$ exposed sides of the final system. Hence:

$$
C=k \frac{\sum P_{n}}{P_{0}}
$$

where $k$ is a constant, can be substituted by unity, but the value of $\frac{1}{4}$ is chosen instead, due to the quadrangle shapes of all components, and to get balance by equal weights of both variables, $\sum P_{n}$ and $P_{0}$, hence:

$$
C=\frac{\sum P_{n}}{4 P_{0}}
$$




\section{Mathematical approach to compactness}

\subsection{Shape factor $\left(S_{f}\right)$ for rectangular shapes}

The geometrical shape of a constant area will be more compact whenever it contracts or shorten and the reverse is true, whenever it elongate or extend, its perimeter will increase and its compactness will decrease. Sides proportion of any rectangle describe its compactness regardless to its area, but to have a general scale capable to describe all shapes compactness, the ratio of perimeter value of a rectangle to a perimeter of a square of equal area can be utilized, which nominated as the shape factor. Assuming a rectangle of area $(A)$, side proportion (r) and shape factor $\left(\mathbf{S}_{\mathbf{f}}\right)$.

The sides of the rectangle will be $\sqrt{A \cdot r}, \sqrt{\frac{A}{r}}$

The perimeter of the rectangle $=2 \sqrt{A \cdot r}+2 \sqrt{\frac{A}{r}}$

The perimeter of a square of similar area $=4 \sqrt{A}$

By definition;

$$
\boldsymbol{S}_{\boldsymbol{f}}=\frac{2 \sqrt{A \cdot r}+2 \sqrt{\frac{A}{r}}}{4 \sqrt{A}}
$$

hence

$$
S_{f}=\frac{r+1}{2 \sqrt{r}}
$$

Table 1: $\quad$ Shape factor values $\left(\boldsymbol{S}_{\boldsymbol{f}}\right)$.

\begin{tabular}{clc}
\hline & $\begin{array}{c}\text { Aspect } \\
\text { Ratio }\end{array}$ & $\begin{array}{c}\text { Shape } \\
\text { Factor }\end{array}$ \\
\hline 1 & $1: 1.0$ & 1.000 \\
\hline 2 & $1: 1.1$ & 1.001 \\
3 & $1: 1.2$ & 1.004 \\
\hline 4 & $1: 1.3$ & 1.009 \\
5 & $1: 1.4$ & 1.014 \\
\hline 6 & $1: 1.5$ & 1.021 \\
7 & $1: 1.6$ & 1.028 \\
\hline 8 & $1: 1.7$ & 1.035 \\
9 & $1: 1.8$ & 1.043 \\
\hline 10 & $1: 1.9$ & 1.052 \\
11 & $1: 2$ & 1.060 \\
\hline 12 & $1: 2.5$ & 1.107 \\
13 & $1: 3$ & 1.155 \\
\hline 14 & $1: 3.5$ & 1.200 \\
15 & $1: 4$ & 1.250 \\
\hline 16 & $1: 4.5$ & 1.296 \\
\hline 17 & $1: 5$ & 1.341 \\
\hline 18 & $1: 10$ & 1.739 \\
19 & $1: 15$ & 2.065 \\
\hline 20 & $1: 20$ & 2.348 \\
\hline 21 & $1: 30$ & 2.830 \\
\hline & & \\
\hline
\end{tabular}


The shape factor is a dimensionless value, having no relation to area, depending only on sides proportion. Hence the shape factor for a square is unity, for a circle is $\left(\frac{\sqrt{\pi}}{2}\right)$. Table 1 indicates values of $\left(\boldsymbol{S}_{\boldsymbol{f}}\right)$ for various side proportion of rectangles.

\subsection{Shape factor $\left(S_{f}\right)$ for three dimensional forms (or form factor)}

Shape factor $\left(\mathbf{S}_{\mathbf{f}}\right)$ for three dimensional forms (or form factor) can be defined as; the ratio of surface area value of a solid to a cube surfaces area value of equal volume. Mahdavi [11] have derived an equation for measuring this factor:

$$
S_{f}=6 \frac{V^{\frac{2}{3}}}{S}
$$

where $V=$ Volume of any solid.

$\mathrm{S}=$ Surface area of the solid.

\subsection{Architectural design compactness $(C)$ for two dimensional shapes}

By definition of the Shape Factor for two dimensional shapes $\left(\boldsymbol{S}_{\boldsymbol{f}}\right)$, the perimeter of a single rectangle is:

$$
P=4 \sqrt{A} \cdot S_{f}
$$

where $P$ is the perimeter of any rectangle.

$A$ its area.

$\boldsymbol{S}_{\boldsymbol{f}}$ its shape factor.

the summation of perimeters of a detached group of rectangles is

$$
\sum P_{n}=4 \sum\left(\sqrt{A_{n}} \cdot \boldsymbol{S}_{\boldsymbol{f n}}\right)
$$

where $\sum\left(\sqrt{A_{n}} \cdot \boldsymbol{S}_{\boldsymbol{f} \boldsymbol{n}}\right)=\sqrt{A_{1}} \cdot \boldsymbol{S}_{\boldsymbol{f} \mathbf{1}}+\sqrt{A_{2}} \cdot \boldsymbol{S}_{\boldsymbol{f} \mathbf{2}}+\cdots \sqrt{A_{n}} \cdot \boldsymbol{S}_{\boldsymbol{f n}}$

But by definition of design compactness,

$$
C=\frac{\sum P_{n}}{4 P_{0}}
$$

Substituting the values $\sum P_{n}$ in eqn.(9) by its value in eqn.(8) we get

$$
C=\frac{\sum\left(\sqrt{A_{n}} \cdot s_{f n}\right)}{P_{o}}
$$

which means that the design compactness equal to the summation of the square root of each space area in a system multiplied by their corresponding shape factors divided by the sum of the exposed sides of the form. Mathematical derivation reveals that shape factor of cells is one of the variables that design compactness varies according to, in addition to the independent variable representing the number of building spaces that a shape consists of.

Average value of design compactness may equal unity which represent a well compact design, less compact design can have lower value, down to 0.25 which represent a detached single square, or even less values, at the same time it can have values more than unity for a very well compact design. 


\section{Results: discussing the compactness of design systems}

In order to compare the compactness of some usual building systems such as linear, square and the courtyard types, a model of abstract plans is prepared, consisting of square units representing the spaces as shown in fig. 7, their compactness were calculated and showed the following results which can be used as guidelines for designers:

1. For continuous linear systems which represent attached housing:

(a) For systems of two units depth, and spaces of depth equal to the external side, fig. 7A. The compactness value will be the unity as follows

$$
C=\frac{n \sqrt{l^{2}}}{n l}=1
$$

(b) If the depth of the attached house is three spaces, fig. 7B, the compactness will be

$$
C=\frac{3 n \sqrt{l^{2}}}{2 n l}=1.5
$$

(c) For a similar system as in 1(a) but with a ratio of space depth to the external side of (r), fig.7C, compactness will be

$$
C=\frac{n \sqrt{r l^{2}}}{n l}=\sqrt{r}
$$

which means that compactness will equal the square roots of the aspect ratio.

2. For continuous linear systems consisting of double loaded corridor which represent office buildings as an example;

(a) If the corridor width is $1 / 3$ of the spaces width, and the building length is nine units, fig.7D, the compactness will be

$$
C=\frac{18 \sqrt{l^{2}}+2.25 * \sqrt{\frac{9 l^{2}}{3}}}{22.66 l}=0.97
$$

(b) For a similar system as in 2(a) but if the aspect ratio of the spaces is (r) fig. 7E, the compactness will be

$$
C=\frac{18 \sqrt{l^{2} * r}+2.25 * \sqrt{\frac{9 l^{2} * r}{3}}}{22.66 l}=0.97 * \sqrt{r}
$$

which means that if the aspect ratio is 1.5 as an example the compactness will be 1.19 . 


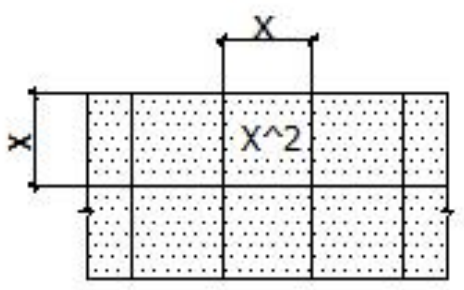

A
B
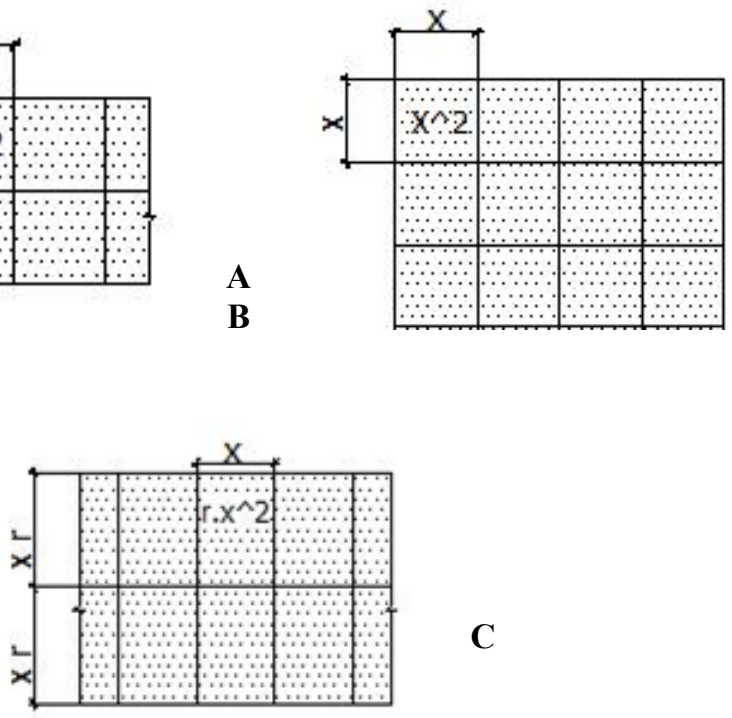

C
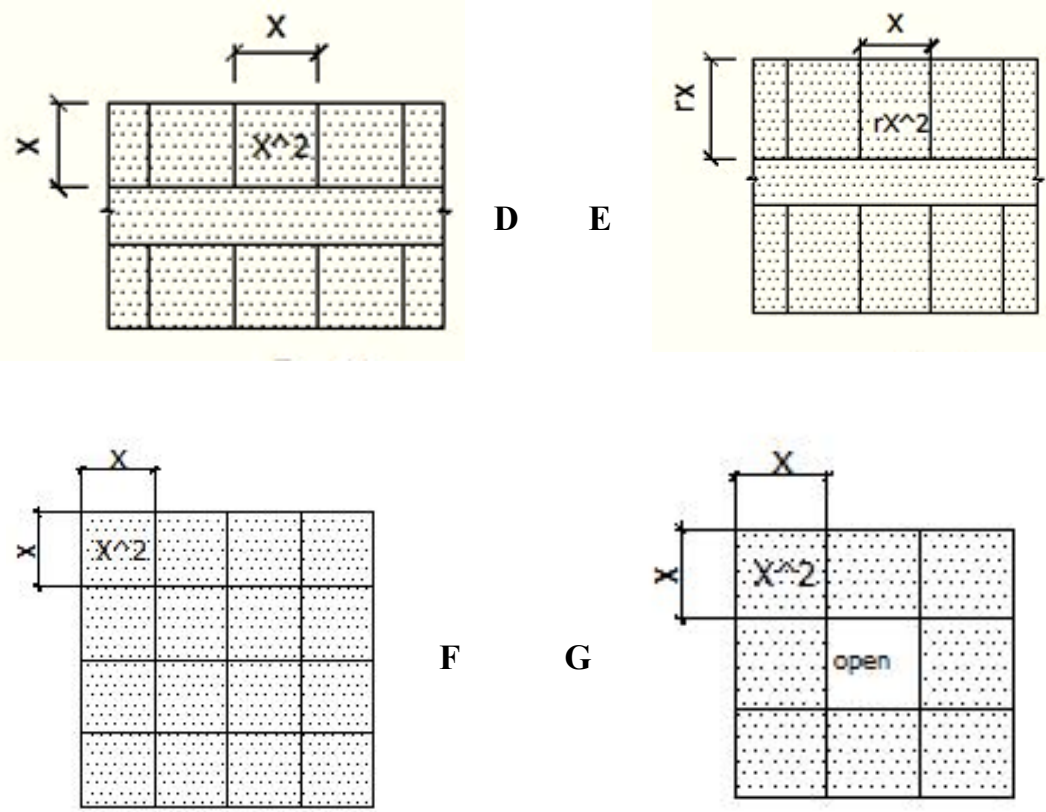

Figure 7: A model of abstract plans consisting of square units representing the spaces in an architectural abstract plan. 
3. For square form building consisting of $(n)$ number of square spaces

(a) If the building is detached, fig.7F, compactness will equal the square root of the number of the spaces divided by four. And it will increase whenever the number of spaces increase.

$$
C=\frac{n \sqrt{A}}{4 \sqrt{A} \sqrt{n}}=\frac{\sqrt{n}}{4}
$$

(b) For a system similar to 3(a) but attached from three sides and with an internal courtyards representing formal housing, fig. $7 \mathrm{G}$ assuming to consist of nine spaces $(3 * 3)$ eight of them indoor and one courtyard space, compactness will equal:

$$
C=\frac{8 \sqrt{A}}{7 \sqrt{A}}=1.14
$$

It is concluded that these systems can result in high values of compactness.

\section{Conclusion}

1. This paper distinguishes between two different scales; shape factor and architectural design compactness. shape factor can describe compactness of abstract (2D) geometrical shapes, while architectural design compactness measures the joining and attachment of the sides of the spaces of a system. The equations, for evaluating both scales are derived.

2. The design compactness scale can be the complementary aspect to the evaluation of heat resistance of building components for controlling heat loss and gain in buildings regardless to the building area, loosed design should have higher values of heat insulations.

3. The derived equation enables computing the external exposed sides of a building before the design stage, depending on the building program and the assumed compactness.

4. Compactness for any design system can be calculated using the method explained in section 4 of this paper, before the design stage to choose the appropriate one.

5. The range of the equation can be extended - in a consequent paper - to cover the design compactness of three dimensional volumes.

\section{References}

[1] Blackwell, W. Area-perimeter ratios (chapter one), Time Saver Standard for architectural design data, Callender, J.H. pp. 72-74 (1982).

[2] Evans, M. Housing, Climate and comfort, The Architectural Press- London, pp. 37.

[3] Burberry, P.Environment and Services, BT Batsford Limited London, p. 82.

[4] Vandenberg, Maritz, AJ Handbook for Building Enclosure, pp. 3135. (1986). 
[5] Lechner, N. Heating, Cooling and Lighting Design methods for architects, John Wiley \& Sons Inc. pp. 441. (2001).

[6] Saeed, M.M. A scale for compactness. Proceeding of the $5^{\text {th }}$ Iraqi Technological Conference of the University of Technology, Iraq (1999).

[7] http://en.wikipedia.org/wiki/Compactness_measure_of_a_shape

[8] Andreas W., Mathias W., Sabine F. web-based metho $\bar{d}$ to generate specific energy consumption data for the evaluation and optimization of building operation. University Karlsruhe (TH) - Department of Architecture. Building Physics and Technical Building Services.

[9] Yurij A. M., Mark C., Cliff M. Forty-Percent Savings and Beyond -Recent Advances in Code Implementation and Development of SuperEfficient Buildings in Russia and Other CIS Countries Center for Energy Efficiency and Research Institute for Building Physics. Institute for Market Transformation.

[10] Zuzana, SVUPS-Nova Slovak republic Implementation of the EPBD in Slovak Republic (2007).

[11] Mahdavi, A. Computational building models: theme and four variations. Eighth International IBPSA Conference. Eindhoven, Netherlands Department of Building Physics and Human Ecology. Vienna University of Technology A-1040, Vienna - Austria. (2003). 